\title{
Phase Lag of Classical Cepheids and RR Lyrae Stars
}

\author{
W. Ogłoza, P. Moskalik \\ Copernicus Astron. Center, ul. Bartycka 18, 00-716 Warsaw, Poland
}

S. Kanbur

Department of Physics $\&$ Astronomy, University of Massachusetts, Amherst, $M A$, USA

\begin{abstract}
It is a well-known property of Cepheids and RR Lyrae variables that their maximum brightness approximately coincides with the maximum expansion velocity. A more detailed investigation (Carson \& Stothers 1984; Simon 1984) has shown that the velocity curve is in fact slightly retarded with the respect to the light variations. In this report we discuss the trends in the observed phase lag for an extensive sample of classical Cepheids and RRab variables.
\end{abstract}

We have collected from the literature simultaneous Johnson $V$ photometry and radial velocity data for 62 fundamental mode and 25 overtone Cepheids. Additional data for 33 fundamental mode RR Lyrae variables has been made available to us by G. Kovács (private communication). Following Simon (1984), we use the Fourier decomposition to define the first order phase lag, $\Delta \phi_{1}$. To that effect, we fit the observed light and velocity curves with the Fourier sum and then define the phase lag $\Delta \phi_{1}=\phi_{1}^{\mathrm{r}_{\mathrm{r}}}-\phi_{1}^{\mathrm{mag}}$. In Fig. 1a we plot $\Delta \phi_{1}$ vs. pulsation period for our classical Cepheid sample. Fundamental mode and overtone Cepheids are displayed with filled and open circles, respectively. The separation of Cepheids into fundamental mode and overtone subgroups is based on the Fourier parameters of their light and velocity curves (Poretti 1994, and references therein; Kienzle et al. 1999) and does not rely on $\Delta \phi_{1}$ in any way. In Fig. 1b the same data is plotted vs, photometric Fourier amplitude $\mathrm{A}_{1}$.

The main results of our analysis can be summarized as follows:

- For all stars $\Delta \phi_{1}$ is negative. This corresponds to maximum expansion velocity occurring after the phase of maximum brightness. Observed behavior is in agreement with the results of hydrodynamical modeling (Simon \& Davis 1983; Antonello \& Aikawa 1995).

- For the fundamental mode Cepheids $\Delta \phi_{1}$ is independent of the pulsation period, with the average value of -0.28 .

- For the overtone Cepheids, $\Delta \phi_{1}$ is lower than for fundamental mode Cepheids of the same period and decreases with period, from -0.24 to -0.71 . The trend is well represented by a linear regression: $\Delta \phi_{1}=-0.983( \pm 0.090) \log P+$ $0.037( \pm 0.047)$.

- For Cepheids with $P>3.5$ day the first-order phase lag $\Delta \phi_{1}$ is a good indicator of the pulsation mode. This property may be particularly useful for identifying 

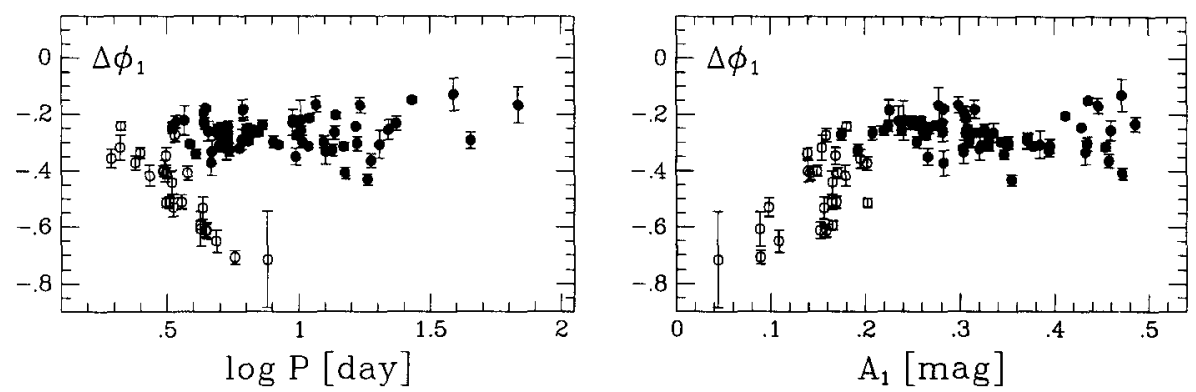

Figure 1. Phase lag $\Delta \phi_{1}$ for classical Cepheids versus a) pulsation period, b) pulsation amplitude. Fundamental mode and overtone pulsators are marked with filled and open circles, respectively.

new long-period overtone Cepheids, for which the criteria based on the light curve shape are no longer effective.

- Neither the $\omega_{2}=2 \omega_{0}$ resonance at $P \approx 10$ day in the fundamental mode Cepheids, nor the $\omega_{4}=2 \omega_{1}$ resonance at $P \approx 4.6$ day in the overtone Cepheids (Kienzle et al. 1999) have any effect on $\Delta \phi_{1}$.

- For the fundamental mode Cepheids $\Delta \phi_{1}$ is independent of the pulsation amplitude.

- For the overtone Cepheids $\Delta \phi_{1}$ correlates with the pulsation amplitude $A_{1}$. This is a mere coincidence, however, resulting from the decrease of the sCepheid amplitude with the pulsation period (Kienzle et al. 1999). Indeed, two very small amplitude s-Cepheids, BG Cru and V1726 Cyg, have almost the same phase lag as their high amplitude siblings of similar periods. Also, in the case of Polaris $\Delta \phi_{1}$ has stayed constant, despite the dramatic decrease of pulsation amplitude over the years (Moskalik \& Ogłoza 2000).

- The phase lag properties of the fundamental mode RR Lyrae variables (RRab stars) are very similar to those of the fundamental mode Cepheids: $\Delta \phi_{1}$ is independent of the pulsation period or pulsation amplitude, with the average value of -0.19 . Because only two RRc stars have been analyzed (TV Boo and $\mathrm{YZ}$ Cap), we cannot say anything about phase lag trends in the group of overtone RR Lyrae pulsators.

\section{References}

Antonello, E. \& Aikawa, T. 1995, A\&A, 302, 105

Carson, T. R. \& Stothers 1984, ApJ, 276, 593

Kienzle, F., Moskalik, P., Bersier, D., \& Pont, F. 1999, A\&A, 341, 818

Moskalik, P. \& Ogłoza, W. 2000, in these proceedings, p. 237

Poretti, E. 1994, A\&A, 285, 524

Simon, N. R. 1984, ApJ, 284, 278

Simon, N. R. \& Davis, C. G. 1983, ApJ, 266, 787 